Supporting Information (SI)

\title{
Fabrication of pH-Adjusted Boronic Acid-Aptamer Conjugate for Electrochemical Analysis of Conjugated $N$-Glycolylneuraminic Acid
}

Lihong Su, ${ }^{\dagger}{ }^{\S}$ Tingjun Chen, ${ }^{\dagger}{ }^{\S}$ Tianxiang Xue, ${ }^{\dagger}$ Anzhi Sheng, ${ }^{\dagger, \dagger}$ Liangfen Cheng ${ }^{\dagger}$ and Juan Zhang ${ }^{*}, \dagger$

'Laboratory of Biosensing Technology, School of Life Sciences, Shanghai University, Shanghai 200444, P. R.

China

¥ Shanghai Key Laboratory of Bio-Energy Crops, Shanghai University, Shanghai 200444, P. R. China

*Corresponding Authors: Juan Zhang

E-mail: juanzhang@shu.edu.cn

Telephone: +86-21-66137037, Fax: +86-21-66137541

${ }^{\S}$ L.S. and T.C. contributed equally to this paper.

Table of Contents

1 EXPERIMENTAL SECTION

1.1 Construction of the boronic acid-aptamer conjugate (BAAC)

1.2 Calibration curve for the measurement of Neu5Gc and Neu5Ac

1.3 Preparation of CNeu5Gc and CNeu5Ac

1.4 Characterization of BAAC, CNeu5Gc, and CNeu5Ac

1.5 Preparation and characterization of $p \mathrm{SC}_{4}-\mathrm{AgNPs}$

1.6 Fluorescent image for the binding of aptamer with CNeu5Gc

2 RESULTS AND DISCUSSION

2.1 Characterization of the boronic acid-aptamer conjugate 
$1 \quad 2.2$ Calibration curve for the measurement of Neu5Ac and Neu5Gc

$2 \quad 2.3$ Characterization of conjugated Neu5Gc and Neu5Ac

$3 \quad 2.4$ Characterization of $\mathrm{pSC}_{4}-\mathrm{AgNPs}$

$4 \quad 2.5$ Linear relationship between $\mathrm{CNeu5Gc}$ and $p \mathrm{SC}_{4}-\mathrm{AgNPs}$

$5 \quad 2.6$ Fluorescent image for the binding of the aptamer with CNeu5Gc

$6 \quad$ 2.7 Cyclic voltammograms and electrochemical impedance spectra for the gold electrode at

7 different modification stages

$8 \quad 2.8$ Capture of Neu5Gc from the mixture of Neu5Gc and Neu5Ac by the aptamer

92.9 Linear relationship between the peak current values and logarithmic values of CNeu5Gc

10 concentrations

112.10 Voltammograms for the detection of different concentrations of CNeu5Gc in real 12 samples. 


\section{EXPERIMENTAL SECTION}

\subsection{Construction of the boronic acid-aptamer conjugate (BAAC)}

EDC $(1.95 \mathrm{mg})$ and NHS $(7.05 \mathrm{mg})$ were dissolved in $100 \mu \mathrm{L}$ deionized water. The mixed solution $(20 \mu \mathrm{L})$ was added to $40 \mu \mathrm{L} \mathrm{CPBA}(500 \mu \mathrm{M})$ in anhydrous alcohol. After 30 minutes, 40 $\mu \mathrm{L}$ of $50 \mu \mathrm{M}$ modified aptamer $\left(\mathrm{NH}_{2}\right.$-AAAAAAAAAATGGTCATGCCGTACGGTGTACCCCC GGGTGTACGCGGTGTACGGCTACTTTCTCTGCGTGCTGAGGGTGATCGTTTTCGCAAA AAAAA-SH) ${ }^{1}$ was added into the mixture and incubated for $3 \mathrm{~h}$. The reaction mixture was purified through a gel column (illustra MicroSpin G-25 column) to give the final product, i.e., boronic acid-aptamer conjugate (BAAC), which was stored at $4{ }^{\circ} \mathrm{C}$.

\subsection{Calibration curve for the measurement of Neu5Gc and Neu5Ac}

The DMB derivative solution was prepared with $8 \mathrm{mM}$ DMB, $1.5 \mathrm{M}$ glacial acetic acid, 0.25 $\mathrm{M}$ sodium thiosulfate, $0.25 \mathrm{M}$ sodium sulfite, and $0.8 \mathrm{mM}$ 2-mercaptoethanol. ${ }^{2}$ Neu5Gc or Neu5Ac standard solution $(90 \mu \mathrm{L})$ with different concentrations were separately mixed with 10 $\mu \mathrm{L}$ DMB derivative solution at $50{ }^{\circ} \mathrm{C}$ for $150 \mathrm{~min}$. The products were analyzed through HPLC with methanol-acetonitrile-ultrapure water $(7: 8: 85)$ as mobile phase, $0.9 \mathrm{~mL} / \mathrm{min}$ of flowing rate, $5 \mu \mathrm{L}$ of injection volume, $30{ }^{\circ} \mathrm{C}$ of column temperature, $373 \mathrm{~nm}$ of the excitation wavelength, and $448 \mathrm{~nm}$ of emission wavelength. The average peak area was calculated for three tests.

\subsection{Preparation of CNeu5Gc and CNeu5Ac}

Neu5Gc or Neu5Ac $(1 \mathrm{mM})$ was dissolved in the solution containing $10 \mathrm{mM}$ EDC and 2.5 mM NHS at $37{ }^{\circ} \mathrm{C}$ for 30 minutes. Then BSA $(20 \mu \mathrm{M})$ was separately added into two kinds of mixed solution and incubated for $3 \mathrm{~h}$, followed by purification through a G-25 agarose gel column to give conjugated Neu5Gc (CNeu5Gc) or conjugated Neu5Ac (CNeu5Ac).

\subsection{Characterization of BAAC, CNeu5Gc, and CNeu5Ac}

BAAC, CNeu5Gc and CNeu5Ac were characterized by the VERTEX 70 Fourier transform infrared spectroscopy (Vertex 70, Bruker Co. Ltd., Bergisch Gladbach, Germany). The measurements were conducted from $4000 \mathrm{~cm}^{-1}$ to $400 \mathrm{~cm}^{-1}$ with $0.2 \mathrm{~cm}^{-1}$ of resolution at room temperature. The obtained spectra were calibrated by OPUS/IR analysis software.

\subsection{Preparation and characterization of $p \mathrm{SC}_{4}-\mathrm{AgNPs}$}

para-Sulfonatocalix[4]arene modified silver nanoparticles $\left(\mathrm{pSC}_{4}-\mathrm{AgNPs}\right)$ were prepared by reduction with sodium borohydride according to the previously reported methods. ${ }^{3,4} 98 \mathrm{~mL}$ $\mathrm{AgNO}_{3}$ aqueous solution $\left(2.04 \times 10^{-4} \mathrm{M}\right)$ and $2 \mathrm{~mL} p \mathrm{SC}_{4}$ aqueous solution $\left(10^{-2} \mathrm{M}\right)$ were mixed 
and stirred in dark for 20 minutes. Then $8.8 \mathrm{mg} \mathrm{NaBH}_{4}$ was added rapidly and stirred continuously at room temperature. After 10 minutes, the mixture was centrifuged at $10000 \mathrm{rpm}$ for 10 minutes. The precipitate was then dispersed in deionized water to obtain $p \mathrm{SC}_{4}-\mathrm{AgNPs}$, which was stored in a brown bottle and stored at $4{ }^{\circ} \mathrm{C}$. The synthesized $p \mathrm{SC}_{4}-\mathrm{AgNPs}$ were characterized by cyclic voltammetry, ultraviolet-visible spectroscopy, VERTEX 70 Fourier transform infrared spectroscopy and dynamic lighting scattering.

\subsection{Fluorescent image for the binding of aptamer with CNeu5Gc}

$50 \mu \mathrm{L}$ modified complementary chain (GCTCAGCACGCACAG-FAM) $(2.4 \mu \mathrm{M})$ was added into $50 \mu \mathrm{L}$ modified aptamer $\left(\mathrm{NH}_{2}\right.$-AAAAAAAAAAAATGGTCCCATGTACGCCGCGGT GTACCTCCGCGTGTACGCTACTTTCTGTGCGTGCTGAGCGTGAATTCGCAAAAAAAA AAAA-Biotin) $(2.4 \mu \mathrm{M})$. The mixture was annealed at $95{ }^{\circ} \mathrm{C}$ for $5 \mathrm{~min}$ and then cooled naturally at room temperature to give the mixed solution of the complementary chain and aptamer $(\mathrm{C}-\mathrm{A})$. Then, $50 \mu \mathrm{L} \mathrm{C}-\mathrm{A}$ was mixed with $50 \mu \mathrm{L}$ immunological magnetic beads (IMB) $(1 \mathrm{mg} / \mathrm{mL})$ at $30{ }^{\circ} \mathrm{C}$ for $3 \mathrm{~h}$, to give C-A/IMB. Subsequently, $100 \mu \mathrm{L}$ CNeu5Gc $(360.396 \mu \mathrm{g} / \mathrm{mL})$ was added into $\mathrm{C}-\mathrm{A} / \mathrm{IMB}$ and the reaction was conducted for $1 \mathrm{~h}$ at $\mathrm{pH} 6$ and $\mathrm{pH} 9$, respectively. After magnetic separation, the modified magnetic beads were analyzed by using fluorescence microscopy.

\section{RESULTS AND DISCUSSION}

2.1 Characterization of the boronic acid-aptamer conjugate

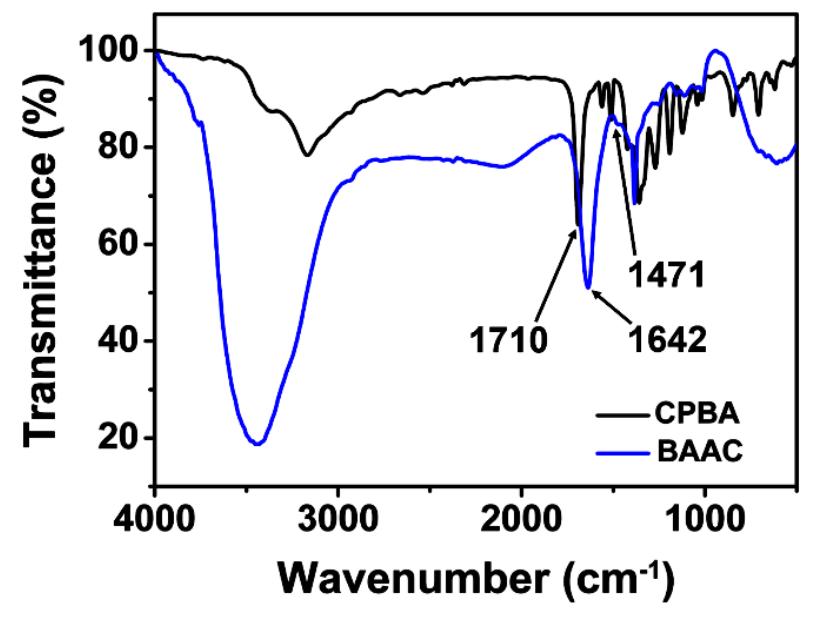

Figure S1. FT-IR spectra of CPBA (black curve) and BAAC (blue curve).

FT-IR spectra of CPBA and BAAC are exhibited in Figure S1. For CPBA, the peak at 1710 
$1 \mathrm{~cm}^{-1}$ can be attributed to stretching vibration of $\mathrm{C}=\mathrm{O}$ in the carboxylic acid moiety. The peak

2 shows an obvious blue shift to $1642 \mathrm{~cm}^{-1}$, as a result of the formation of amide bond for BAAC.

3 Meanwhile, the appearance of new absorption band at $1471 \mathrm{~cm}^{-1}$ can be contributed to stretching

4 vibration of $\mathrm{C}-\mathrm{N}$ in amide segment of BAAC. These results well signify the successful

5 occurrence of amide bond.

\subsection{Calibration curve for the measurement of Neu5Ac and Neu5Gc}
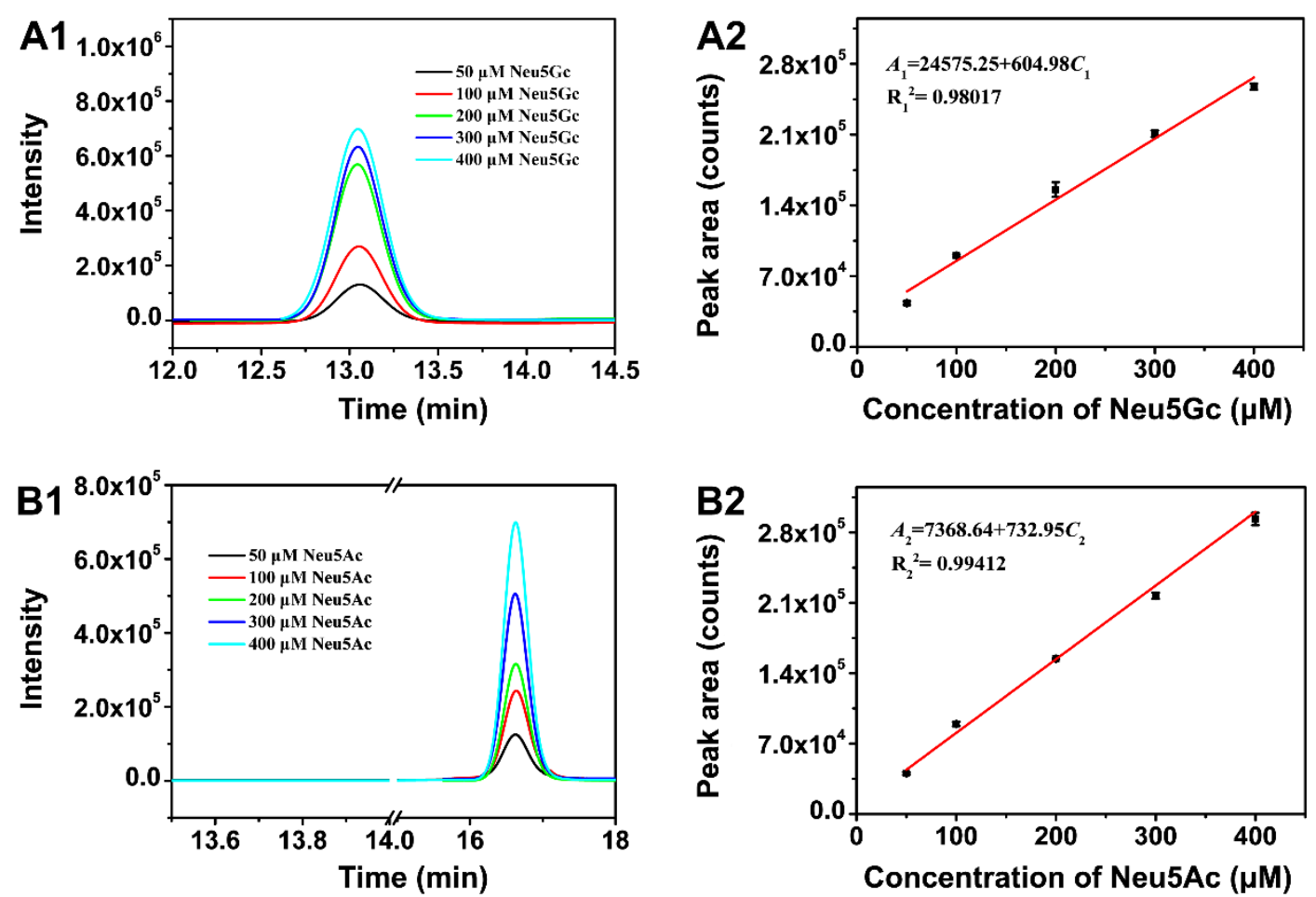

Figure S2. (A1) High performance liquid chromatograms of Neu5Gc with different concentrations. (A2) The linear relationship between peak area (counts) and Neu5Gc concentrations. (B1) High performance liquid chromatograms of Neu5Ac with different concentrations. (B2) The linear relationship between peak area (counts) and Neu5Ac concentrations.

Figure S2 (A1) and (A2) exhibit high performance liquid chromatograms of different concentrations of Neu5Gc and Neu5Ac with retention time of 13.083 min and $16.625 \mathrm{~min}$, respectively. The peak area values linearly increase with the increased concentrations of Neu5Gc or Neu5Ac from 50 to $400 \mu \mathrm{M}$ (Figure S2 (A2) and (B2)). For Neu5Gc, the linear fitting equation of $A_{l}=24575.25+604.98 C_{l}(\mu \mathrm{M})$ can be given with a correlation coefficient of 
10.98017 , where $A_{1}$ is the peak area and $C_{l}$ is Neu5Gc concentration (Figure S2 (A2)). For

2 Neu5Ac, the linear fitting equation of $A_{2}=7368.64+732.95 C_{2}(\mu \mathrm{M})$ can be obtained with a

3 correlation coefficient of 0.99412 , in which $A_{2}$ is the peak area of Neu5Ac and $C_{2}$ is the

4 concentration of Neu5Ac (Figure S2 (B2)).

$5 \quad 2.3$ Characterization of conjugated Neu5Gc and Neu5Ac

6

7
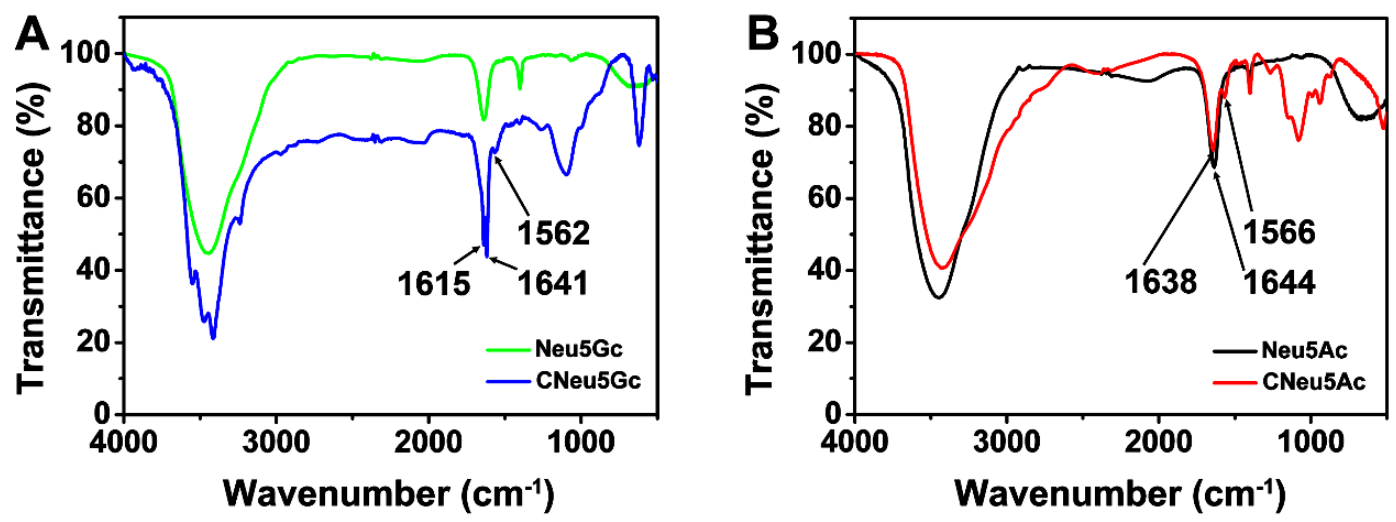

Figure S3. (A) FT-IR spectra of Neu5Gc (green curve) and CNeu5Gc (blue curve). (B) FT-IR spectra of Neu5Ac (black curve) and CNeu5Ac (red curve).

The prepared CNeu5Gc and CNeu5Ac have been characterized by the FT-IR spectra and the corresponding results have been given in Figure S3. For CNeu5Gc, two obviously strong peaks at $1641 \mathrm{~cm}^{-1}$ and $1615 \mathrm{~cm}^{-1}$ can be separately contributed to stretching vibration of $\mathrm{C}=\mathrm{O}$ (amide I) and bending vibration of $\mathrm{N}-\mathrm{H}$ (amide II) (Figure S3 (A)). In the meantime, the peak at $1562 \mathrm{~cm}^{-1}$ can be ascribed to stretching vibration of $\mathrm{C}-\mathrm{N}$ (Figure S3 (A)). These results well suggest the successful synthesis of CNeu5Gc. Furthermore, compared with that of Neu5Ac, a new absorption peak at $1566 \mathrm{~cm}^{-1}$ attributed to stretching vibration of $\mathrm{C}-\mathrm{N}$, can be found for CNeu5Ac (Figure S3 (B)). Meanwhile, the vibration peak of $\mathrm{C}=\mathrm{O}$ shifts from $1644 \mathrm{~cm}^{-1}$ to 1638 $\mathrm{cm}^{-1}$ (Figure S3 (B)), confirming the formation of amide bond between carboxylic acid group of Neu5Ac and amine group of BSA. 

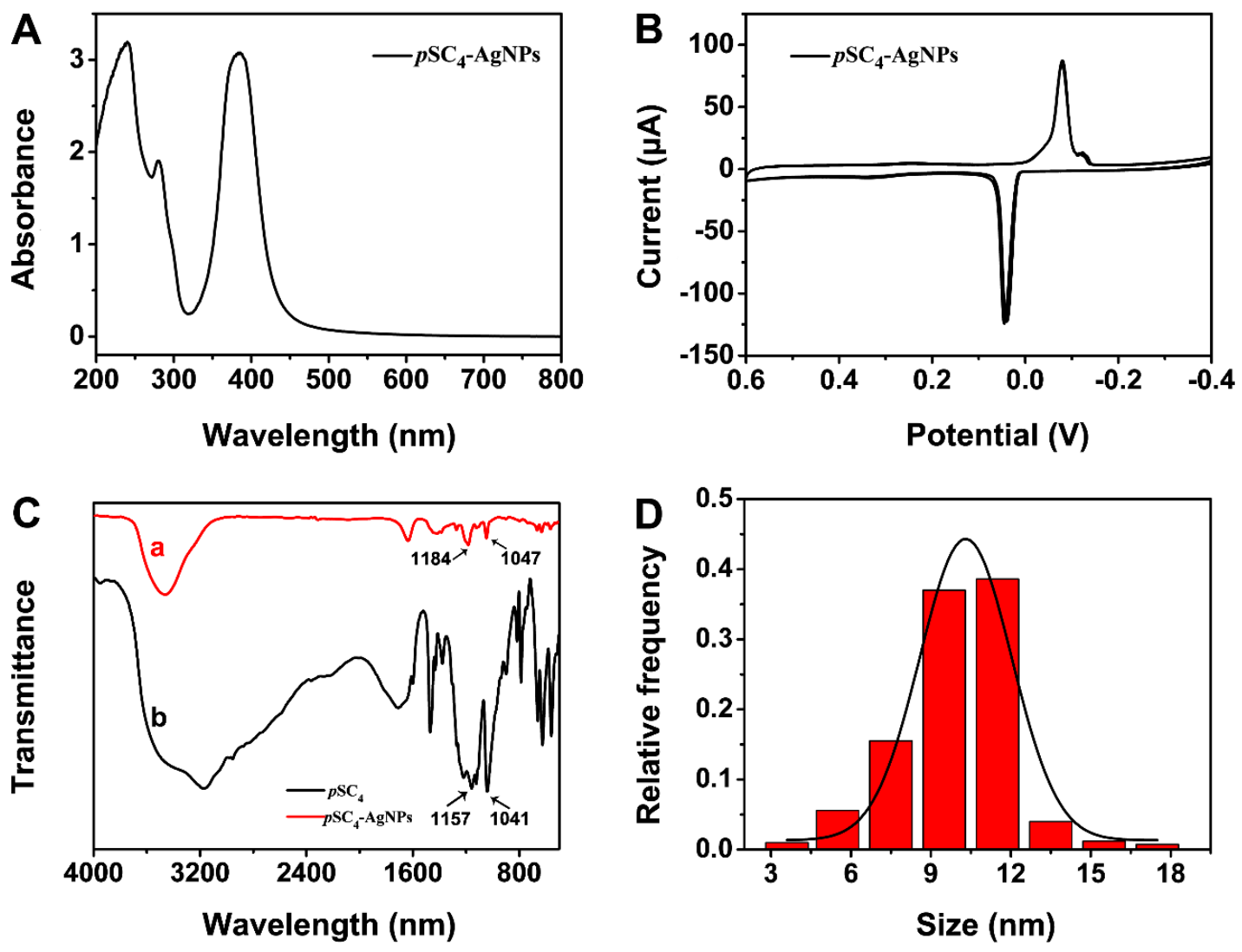

Figure S4. (A) UV-visble spectrum of $\mathrm{pSC}_{4}-\mathrm{AgNPs}$. (B) Cyclic voltammogram of $p \mathrm{SC}_{4}-$ AgNPs. (C) FT-IR spectra of (a) $p \mathrm{SC}_{4}$, (b) $p \mathrm{SC}_{4}-\mathrm{AgNPs}$. (D) The hydration diameter of $p \mathrm{SC}_{4}-\mathrm{AgNPs}$ determined by dynamic lighting scattering.

The synthesized $p \mathrm{SC}_{4}-\mathrm{AgNPs}$ have been characterized by using ultraviolet-visible spectroscopy, cyclic voltammetry, FT-IR spectroscopy and dynamic lighting scattering. As shown in Figure $\mathrm{S} 4(\mathrm{~A})$, an absorption peak at $280 \mathrm{~nm}$ suggests the appearance of $p \mathrm{SC}_{4}$ around AgNPs. ${ }^{5}$ Additionally, the peak at $400 \mathrm{~nm}$ is the characteristic absorption peak for AgNPs. ${ }^{6}$ Meanwhile, a pair of obvious redox peaks can be given (Figure S4 (B)). For $p \mathrm{SC}_{4}-\mathrm{AgNPs}$, the absorption bands between $1070 \mathrm{~cm}^{-1}$ and $1250 \mathrm{~cm}^{-1}$ can be attributed to the symmetric and asymmetric stretching vibrations of $\mathrm{RSO}_{3}$ around $\mathrm{AgNPs}$ (Figure $\mathrm{S} 4(\mathrm{C})$ ). Moreover, an average hydration diameter of $11 \mathrm{~nm}$ can be observed for $p \mathrm{SC}_{4}-\mathrm{AgNPs}$ (Figure $\mathrm{S} 4$ (D)). These results well imply the successful synthesis of $p \mathrm{SC}_{4}-\mathrm{AgNPs}$. 


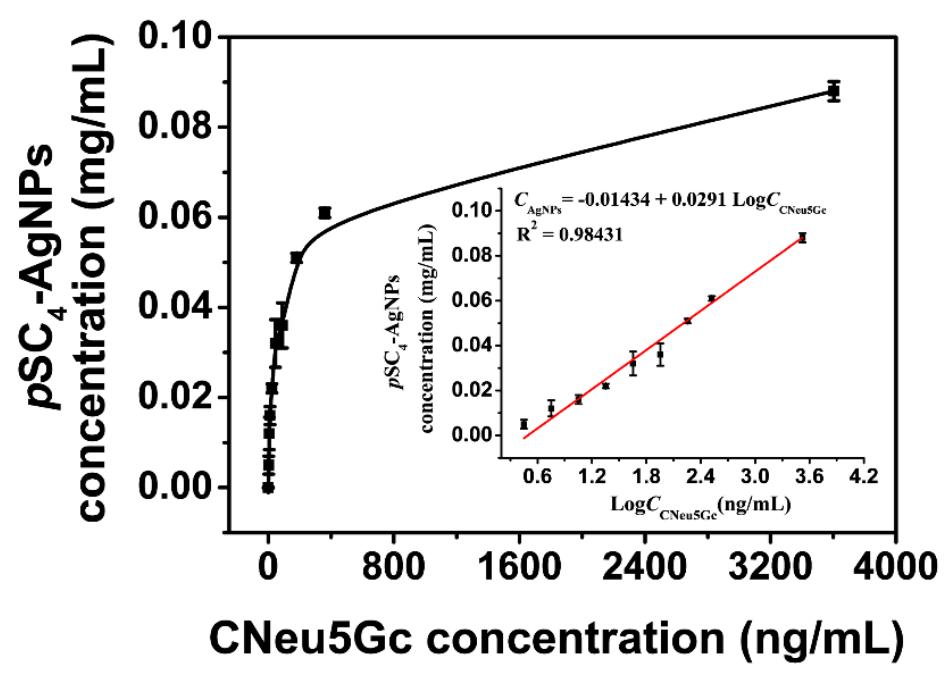

3 Figure S5. $p \mathrm{SC}_{4}-\mathrm{AgNPs}$ concentrations versus different $\mathrm{CNeu} 5 \mathrm{Gc}$ concentrations. Inset: the

4 linear relationship between $p \mathrm{SC}_{4}-\mathrm{AgNPs}$ concentrations and logarithmic values of $\mathrm{CNeu} 5 \mathrm{Gc}$ 5 concentrations.

A linear fitting equation of $C_{\mathrm{AgNPs}}=-0.01434+0.0291 \log C_{\mathrm{CNeu} 5 \mathrm{Gc}}$ can be given with a 7 correlation coefficient of 0.98431 , where $C_{\mathrm{AgNPs}}$ is the concentration of $p \mathrm{SC}_{4}-\mathrm{AgNPs}$ and $8 C_{\mathrm{CNeu} 5 \mathrm{Gc}}$ is the concentration of CNeu5Gc.

2.6 Fluorescent image for the binding of the aptamer with CNeu5Gc

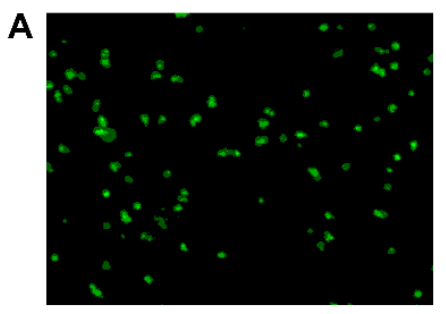

C-A/IMB

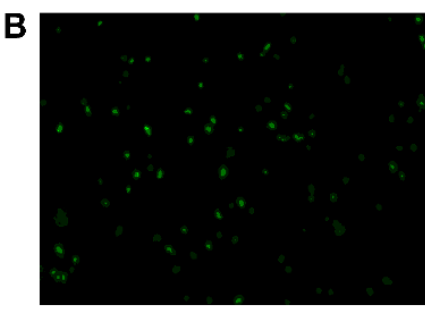

pH 6

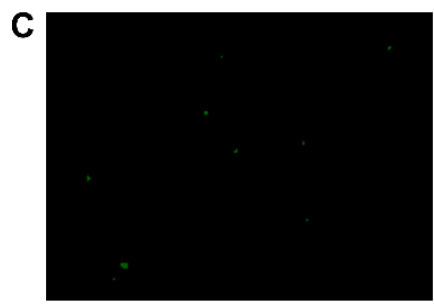

$\mathrm{pH} 9$

Figure S6. (A) Fluorescent image of $\mathrm{C}-\mathrm{A} / \mathrm{IMB}$. Fluorescent image obtained after binding with CNeu5Gc with pH values of (B) 6 and (C) 9.

The obviously green fluorescence can be observed for C-A/IMB (Figure S6 (A)). The decreased fluorescence can be found after the binding of CNeu5Gc at pH 6 (Figure S6 (B)), which can be ascribed for the release of FAM modified complementary strand into the solution. 
Moreover, the weak fluorescence can be found after the binding of $\mathrm{CNeu} 5 \mathrm{Gc}$ at $\mathrm{pH} 9$ (Figure $\mathrm{S} 6$ (C)), suggesting the strong capture capability of aptamer on $\mathrm{CNeu} 5 \mathrm{Gc}$ at $\mathrm{pH} 9$.

2.7 Cyclic voltammograms and electrochemical impedance spectra for the gold electrode at different modification stages
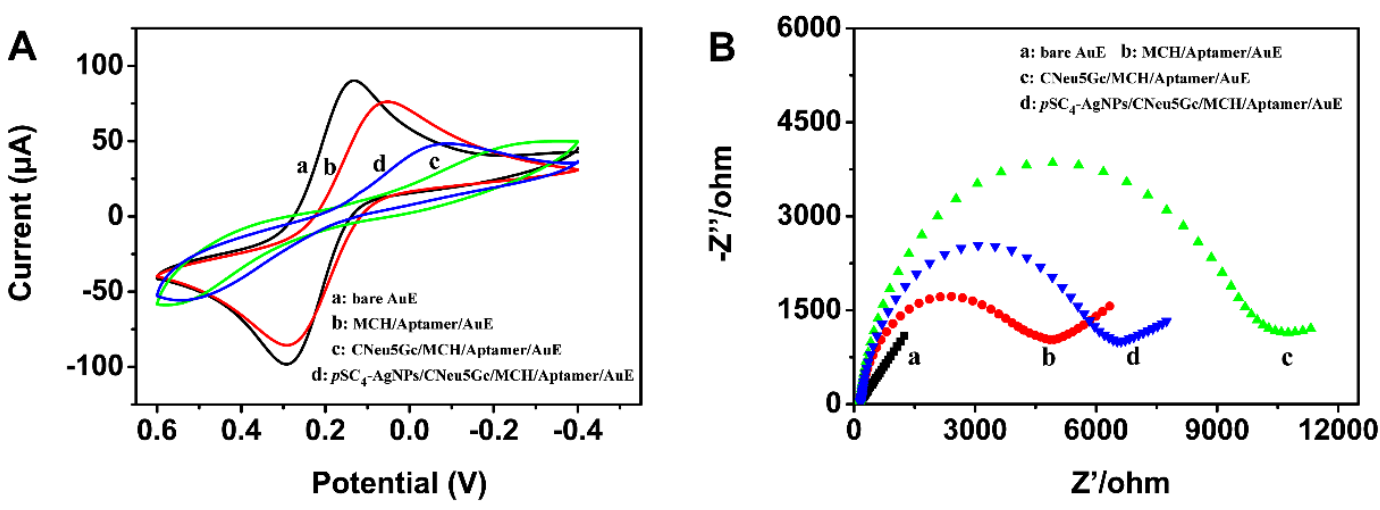

Figure S7. (A) Cyclic voltammograms for gold electrode at different modification stages: (a)
bare AuE,
(b) $\mathrm{MCH} / \mathrm{Aptamer} / \mathrm{AuE}$,
(c) $\mathrm{CNeu} 5 \mathrm{Gc} / \mathrm{MCH} / \mathrm{Aptamer} / \mathrm{AuE}$, and $p \mathrm{SC}_{4}-\mathrm{AgNP} / \mathrm{CNeu} 5 \mathrm{Gc} / \mathrm{MCH} / \mathrm{Aptamer} / \mathrm{AuE}$. (B) Complex plane plot for the electrochemical impedance measurements of gold electrode at different modification stages: (a) bare AuE, (b) MCH/Aptamer/AuE, CNeu5Gc/MCH/Aptamer/AuE, and pSC4-AgNPs/CNeu5Gc/MCH/Aptamer/AuE. Electrolyte: phosphate buffer (5 mM, pH 7.0). Electrochemical species: $5 \mathrm{mM}\left[\mathrm{Fe}(\mathrm{CN})_{6}\right]^{3-14-}$ containing $0.1 \mathrm{M} \mathrm{KCl}$. Biasing potential: $0.224 \mathrm{~V}$. Amplitude: $10 \mathrm{mV}$. Frequency range: $0.01 \mathrm{~Hz}$ to $10 \mathrm{kHz}$.

A reversible redox peak (curve $a$, Figure S7(A)) and almost no semicirclein (curve $a$, Figure S7(B)) can be observed for bare gold electrode. After modification of aptamer and $\mathrm{MCH}$, the semicircle can be detected (curve $b$, Figure S7(B)), indicating an increase in electronic resistance, which can be explained by the insulating nature of aptamer and $\mathrm{MCH}$. CNeu5Gc can be further modified to the electrode surface through aptamer specific recognition and borate bond, further increase of $\mathrm{R}_{\mathrm{et}}$ can be detected (curve $c$, Figure $\mathrm{S} 7(\mathrm{~B})$ ). Meanwhile, the peak becomes irreversible (curve $c$, Figure S7(A)). This is because CNeu5Gc creates a greater barrier on the electrode surface, preventing the redox label $\left[\mathrm{Fe}(\mathrm{CN})_{6}\right]^{3-/ 4-}$ from approaching the electrode surface. When the increased $p \mathrm{SC}_{4}-\mathrm{AgNPs}$ is bound to the surface of $\mathrm{CNeu} 5 \mathrm{Gc}$, a semicircle slightly smaller than CNeu5Gc/MCH/Aptamer/AuE (curve $c$, Figure S7(B)) will be detected 
1 (curve $d$, Figure S7(B)), which can be explained by the fact that the ability of silver nanoparticles

2 to promote conductivity is greater than the insulation effect of $\mathrm{CNeu} 5 \mathrm{Gc}$.
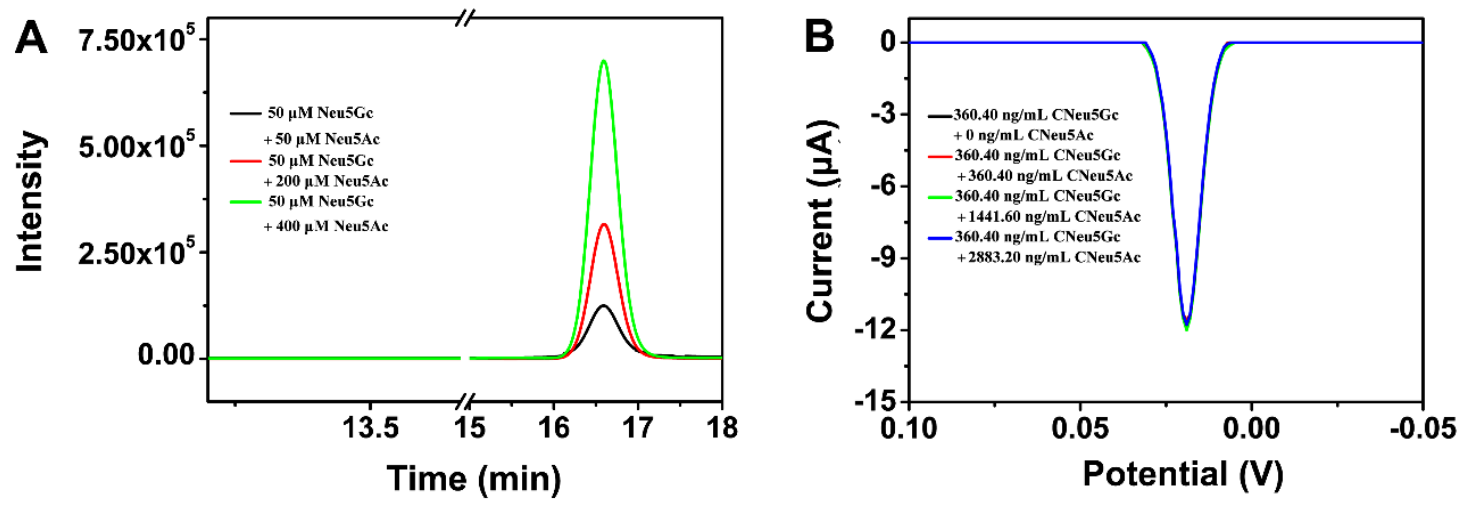

Figure S8. (A) HPLC chromatograms of supernatants obtained after magentic separation. The molar ratios between Neu5Gc and Neu5Ac are 1:1, 1:4 and 1:8. (B) Linear sweep voltammograms for the mixture of $\mathrm{CNeu} 5 \mathrm{Gc}$ and $\mathrm{CNeu} 5 \mathrm{Ac}$ with different molar ratios $(1: 1,1: 4$ 8 and 1:8).

The experiments have been designed and carried out to capture Neu5Gc from the mixture of Neu5Gc and Neu5Ac with the aid of immune magnetic beads, and the corresponding results have been given in Figure S8. When the molar ratio of Neu5Gc and Neu5Ac was changed from 1:1 to 1:8, only peak attributed to Neu5Ac, appears with the increasing peak area (Figure S8 (A)). This can be explained for specific capture of Neu5Gc by the apamer on the surface of magnetic bead, resulting in the exsitence of only Neu5Ac in the supernatant solution after magnetic separation. Accordingly, almost same peak current can be found for the mixed solution of CNeu5Gc and CNeu5Ac with molar ratios of 1:1, 1:4 and 1:8 (Figure S8 (B)). These results well confirm that aptamer has a good ability to capture $\mathrm{CNeu} 5 \mathrm{Gc}$ from the mixture of $\mathrm{CNeu} 5 \mathrm{Gc}$ and CNeu5Ac. 

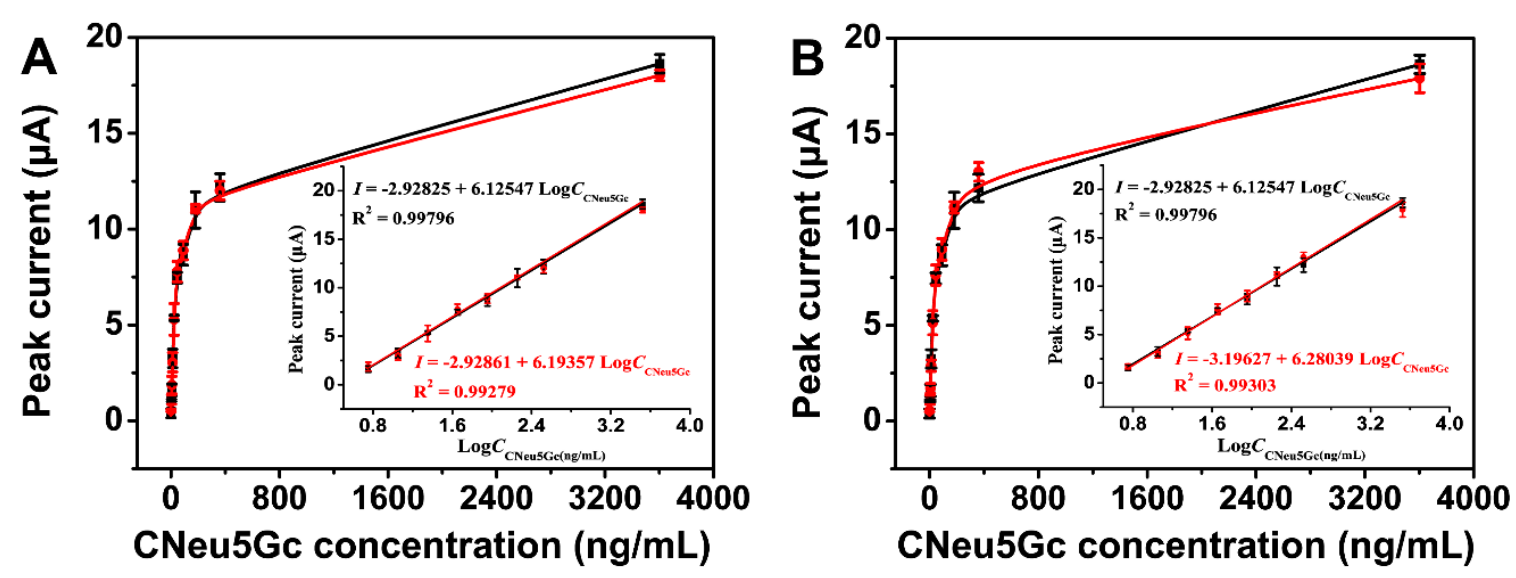

$4 \quad$ Figure S9. (A) Peak current values versus different CNeu5Gc concentrations in buffer (black line)

5 and in the mixture of CNeu5Gc and Neu5Ac with molar ratio of 1:5 (red line). Inset: the linear relationship between peak current values and logarithmic values of CNeu5Gc concentrations in buffer (black line) and in the mixture of CNeu5Gc and Neu5Ac with molar ratio of 1:5 (red line). (B) Peak current values versus different $\mathrm{CNeu} 5 \mathrm{Gc}$ concentrations in buffer (black line) and in the mixture of $\mathrm{CNeu} 5 \mathrm{Gc}$ and $\mathrm{CNeu} 5 \mathrm{Ac}$ with molar ratio of 1:5 (red line). Inset: the linear relationship between peak current values and logarithmic values of CNeu5Gc concentrations in buffer (black line) and in the mixture of CNeu5Gc and CNeu5Ac with molar ratio of 1:5 (red line).

The mathematical relationships between peak current values and different CNeu5Gc concentrations in the mixture of $\mathrm{CNeu} 5 \mathrm{Gc}$ and $\mathrm{Neu} 5 \mathrm{Ac}$ or in the mixture of $\mathrm{CNeu} 5 \mathrm{Gc}$ and CNeu5Ac have been established. As shown in Figure S9 (A), the linear fitting equation of $I=$ $-2.92861+6.19357 \log C_{\mathrm{CNeu} 5 \mathrm{Gc}}$ can be given with a correlation coefficient of 0.99279 , where $I$ is the peak current value and $C_{\mathrm{CNeu} 5 \mathrm{Gc}}$ is the concentration of $\mathrm{CNeu} 5 \mathrm{Gc}$ in the mixed solution of Neu5Gc and Neu5Ac with molar ratio of 1:5. In addition, a linear fitting equation of $I=-3.19627$ $+6.28039 \log C_{\mathrm{CNeu} 5 \mathrm{Gc}}$ can be given for the mixture of CNeu5Gc and CNeu5Ac (Figure S9 (B)). Therefore, the mathematical relationships between peak current values and different concentrations of CNeu5Gc are still feasible when using a mixture of CNeu5Gc and Neu5Ac or a mixture of CNeu5Gc and CNeu5Ac. 

samples
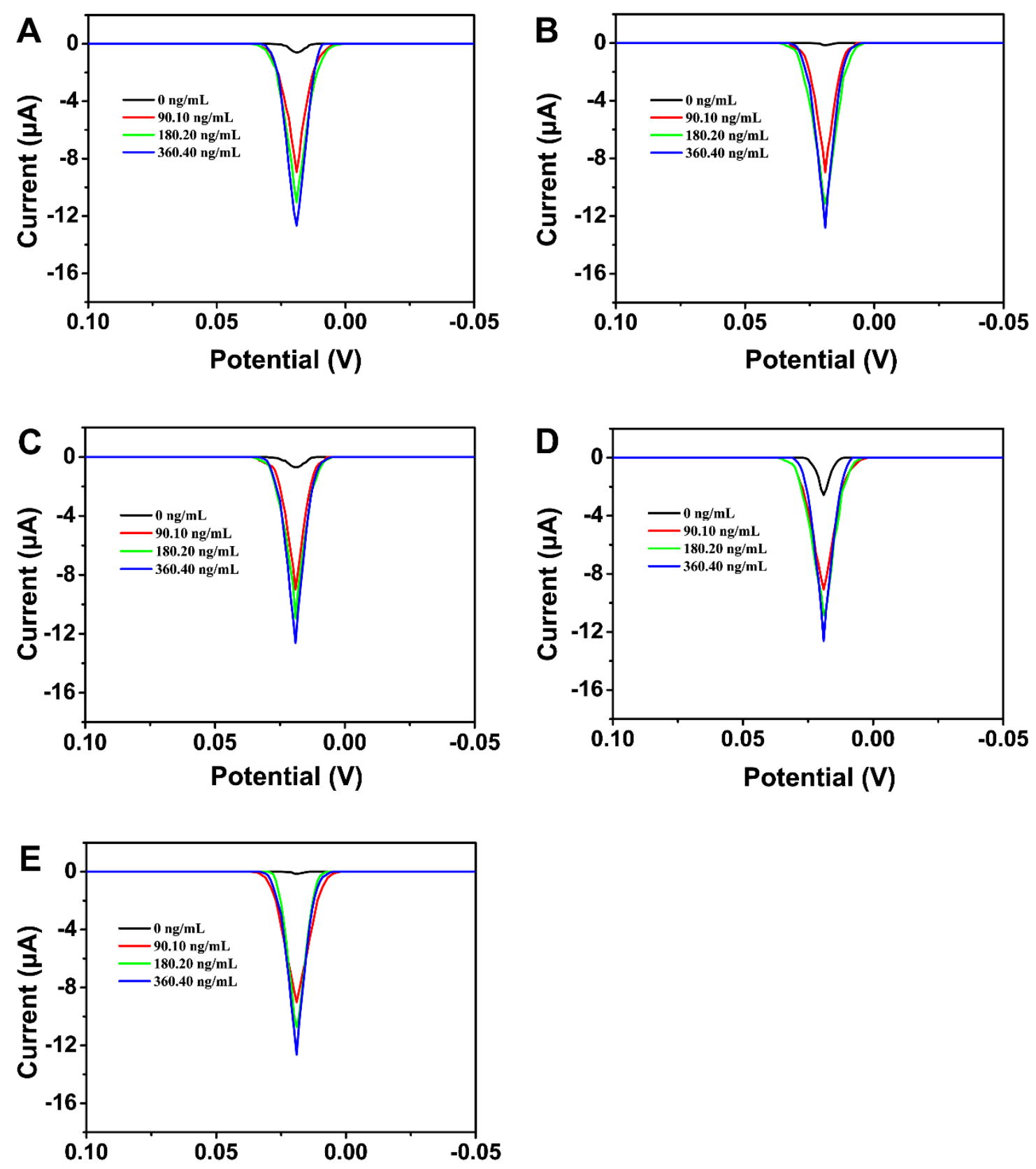

\section{References}

8 (1) Gong, S.; Ren, H. L.; Lin, C.; Hu, P.; Tian, R. Y.; Liu, Z. S.; Li, Y. S.; Zhou, Y.; Yang, Y.; Lu, S. 9 Y. Immunochromatographic Strip Biosensor for the Rapid Detection of N-Glycolylneuraminic 
1 Acid Based on Aptamer-Conjugated Nanoparticle. Anal. Biochem. 2018, 561, 52-58.

2 (2) Hedlund, M.; Padler Karavani, V.; Varki, N. M.; Varki, A. Evidence for a Human-Specific 3 Mechanism for Diet and Antibody-Mediated Inflammation in Carcinoma Progression. Proc. Natl. 4 Acad. Sci. India 2008, 105, 18936-18941.

5 (3) Xiong, D. J.; Li, H. B. Colorimetric Detection of Pesticides Based on Calixarene Modified 6 Silver Nanoparticles in Water. Nanotechnology 2008, 19, 465502-465502.

7 (4) Zhang, J.; Chen, H.; Cao, Y.; Feng, C.; Zhu, X. L.; Li, G. X. Design Nanoprobe Based on its 8 Binding with Amino Acid Residues on Cell Surface and its Application to Electrochemical 9 Analysis of Cells. Anal. Chem. 2018, 91, 1005-1010.

10 (5) Diao, G. W.; Zhou, W. The Electrochemical Behavior of p-Sulfonated Calix [4] Arene. J. 11 Electroanal. Chem. 2004, 567, 325-330.

(6) Rastogi, L.; Sashidhar, R. B; Karunasagar, D.; Arunachalam, J. Gum Kondagogu 13 Reduced/Stabilized Silver Nanoparticles as Direct Colorimetric Sensor for the Sensitive Detection of $\mathrm{Hg}^{2+}$ in Aqueous System. Talanta 2014, 118, 111-117. 